\title{
Duração do onset vocálico da fala fluente de gagos
}

\author{
Voice onset duration of stutterers' fluent speech
}

\author{
Cláudia Fassin Arcuri ${ }^{1}$, Ellen Osborn², Ana Maria Schiefer ${ }^{3}$, Brasília Maria Chiari $^{4}$
}

\begin{abstract}
RESUMO
Objetivo: Comparar a duração da emissão da fala fluente de adultos com diferentes graus de gagueira em tarefa de repetição de frases. Métodos: Seis adultos gagos, com grau de severidade variando de leve a severo, foram pareados com indivíduos sem nenhuma alteração de linguagem. O corpus foi composto pelas palavras "cavalo", "pipoca" e " tapete", inseridas em frases-veículo "Digo...... baixinho". As frases foram emitidas pela pesquisadora e repetidas três vezes pelos indivíduos. A fala foi gravada diretamente em computador, tendo sido desconsideradas as emissões disfluentes. Posteriormente, foram realizadas as medidas de duração acústica das amostras, através do programa Praat 4.3. As frases foram divididas em segmentos delimitados por dois onsets vocálicos consecutivos. Os dados foram tabulados e analisados estatisticamente através do Teste-t e da ANOVA (Analysis of variance). Resultados: Os gagos levaram mais tempo para emitir todas as unidades estudadas, com exceção da unidade [ib]. Na comparação entre os diferentes graus de severidade da gagueira, verificou-se que os gagos leves e moderados apresentam medidas de duração semelhantes e diferentes da dos gagos severos. Conclusão: Os parâmetros acústicos de duração delimitados pela medida dos onset vocálicos demonstraram que os gagos diferem dos não-gagos, além destas medidas diferenciarem os graus de severidade da gagueira.
\end{abstract}

Descritores: Transtornos da articulação; Distúrbios da fala; Gagueira; Acústica da fala; Medida da produção da fala; Espectrografia do som

\section{INTRODUÇÃO}

A gagueira é considerada uma desordem de sincronização do cérebro, uma alteração rítmica, causadora de rupturas na programação dos movimentos musculares exigidos pela fala ${ }^{(1)}$.

Os aspectos linguísticos e motores que caracterizam a patologia tornam a gagueira multidimensional, com uma base genética e neurofuncional. O processamento prosódico é um dos elementos fundamentais para compreender a gagueira, no que diz respeito ao comprometimento rítmico característico da patologia. O sistema motor que controla a fala deve controlar a velocidade articulatória e, qualquer desarranjo implicaria em provável episódio de disfluência ${ }^{(2)}$.

A psicolinguística tem contribuído para o entendimento

Trabalho realizado no Departamento de Fonoaudiologia da Universidade Federal de São Paulo - UNIFESP - São Paulo (SP), Brasil, baseado em dissertação de mestrado apresentada em 2007.

(1) Pós-graduanda (Doutorado) do Programa de Pós-graduação em Distúrbios da Comunicação Humana da Universidade Federal de São Paulo - UNIFESP

- São Paulo (SP), Brasil

(2) Mestre, Professora Adjunto I do Departamento de Fonoaudiologia da Universidade Federal de São Paulo - UNIFESP - São Paulo (SP), Brasil.

(3) Doutora, Professora Associada do Departamento de Fonoaudiologia da Universidade Federal de São Paulo - UNIFESP - São Paulo (SP), Brasil.

(4) Livre-docente, Professora Titular do Departamento de Fonoaudiologia da Universidade Federal de São Paulo - UNIFESP - São Paulo (SP), Brasil. Endereço para correspondência: Cláudia Fassin Arcuri. Av. Camilo Castelo Branco, 160, Vl. Gumercindo, São Paulo (SP), Brasil, CEP: 04130-020. Email: clauarcuri@gmail.com

Recebido em: 30/10/2008; Aceito em: 9/5/2009 da fala, seja ela fluente ou disfluente. Para a emissão de uma mensagem, um mecanismo complexo é acionado e esse mecanismo, para ser eficiente e econômico, trabalha com a memorização de unidades mínimas de número limitado, que podem ser combinadas, gerando infinitas possibilidades de construção, uma vez que seria impossível o armazenamento de toda a informação que se desejaria transmitir ${ }^{(3)}$.

Um dos instrumentos para a compreensão dos mecanismos de fala é a análise acústica, que tornou possível a apreciação das principais características acústicas do espectro de fala de indivíduos normais: a frequência, intensidade e a duração ${ }^{(4)}$, inclusive no Português Brasileiro.

O parâmetro acústico de duração é uma medida objetiva que compõe a análise acústica da voz e da fala e está intimamente relacionada com o ritmo e a taxa de elocução da fala ${ }^{(5)}$. Esta análise tem contribuído para o melhor conhecimento dos padrões e processos que compõem o discurso e suas alterações ${ }^{(6)}$.

A avaliação acústica da fala dos gagos nos possibilita fazer inferências a respeito de aspectos articulatórios e fisiológicos, por vezes diferentes daqueles encontrados na fala dos indivíduos não-gagos. Essas diferenças marcam as perturbações da temporalidade do controle motor da fala presentes no discurso fluente e disfluente do indivíduo que manifesta a gagueira.

Sendo assim, o presente trabalho tem por objetivo comparar a duração da emissão da fala fluente de indivíduos adultos com diferentes graus de gagueira, em tarefa de repetição de frases. 


\section{MÉTODOS}

Para este estudo os procedimentos de seleção foram iniciados após o parecer do Comitê de Ética em Pesquisa da Universidade Federal de São Paulo (UNIFESP), sob protocolo $\mathrm{n}^{\circ} 0159 / 05$. Todos os participantes foram informados a respeito da pesquisa e assinaram termo de consentimento.

Foram selecionados seis indivíduos adultos (com idades entre 22 e 35 anos; média de 26 anos e dois meses) com queixa de gagueira, sendo três homens e três mulheres, atendidos no Ambulatório de Avaliação Fonoaudiológica do Hospital São Paulo da UNIFESP nos anos de 2005 e 2006.

Todos submeteram-se a avaliação fonoaudiológica convencional: anamnese, avaliação da comunicação, avaliação audiológica básica e específica da gagueira.

Para a avaliação específica da gagueira foi realizada gravação de fala espontânea e leitura, com filmadora Digital Sony ${ }^{\circledR}$, em fita MiniDv, conectada a um microfone headset Plantronics ${ }^{\circledR}$, modelo Audio20, em sala acusticamente tratada. As gravações foram transcritas canonicamente e, a seguir, as disfluências foram mapeadas em típicas e atípicas. Foi adotado como critério a presença de, no mínimo, $3 \%$ de disfluências atípicas para estabelecimento do diagnóstico e posterior preenchimento do protocolo específico da gagueira - Stuttering Severity Instrument 3 (SSI-3) ${ }^{(7)}$, para definição do grau de severidade.

Os indivíduos dividiram-se em três grupos conforme o grau de severidade da gagueira: dois apresentaram gagueira de grau leve, dois de grau moderado e dois de grau severo. O grupo controle foi composto por dois indivíduos, um homem e uma mulher, sem queixas de fala e linguagem, alfabetizados, e com idade entre 23 e 30 anos (média de 26 anos e seis meses). Os indivíduos do grupo controle apresentaram menos de $2 \%$ de disfluências atípicas em sua emissão, tendo pontuação inferior a dez no protocolo SSI-3, excluindo-se a possibilidade de eles serem classificados como apresentando gagueira muito leve.

Uma vez que a proposta do estudo era estabelecer medidas de duração que propiciem a comparação entre sujeitos gagos durante emissões fluentes, foi realizada a análise da fala em situação de repetição, uma vez que sob repetição os gagos tendem a apresentar episódios raros de disfluência devido ao modelo previamente oferecido. Foram desconsiderados os trechos de fala onde porventura ocorressem rupturas na emissão.

A partir disso, e visando possibilitar a coleta dos dados, foram selecionadas três palavras trissílabas paroxítonas, iniciadas por consoantes surdas ("pipoca", "cavalo" e "tapete"), que foram inseridas em uma frase-veículo do tipo: "Digo ___ baixinho", assim como ocorreu em outros estudos previamente realizados ${ }^{(5,8)}$. Cada frase era emitida pela pesquisadora e repetida em voz alta pelos indivíduos amostrados sendo que estas foram apresentadas em ordem aleatória, pelo menos três vezes, evitando viés para a análise estatística.

O registro das amostras de fala se deu em sala acusticamente tratada, por meio de gravação em laptop HP Pavillion ${ }^{\circledR}$ ze5500, com microfone do tipo headset Plantronics ${ }^{\circledR}$, modelo Audio20, a uma distância aproximada de $10 \mathrm{~cm}$ da rima da boca do indivíduo, através do software Sound Forge 4.5.

Estudos linguísticos indicam que a produção de um enunciado se dá através de um fluxo vocálico interrompido por perturbações consonantais ${ }^{(5)}$. Deste modo, a unidade delimitada por dois onsets vocálicos consecutivos é denominado na literatura de unidade vogal-vogal (VV). Com base neste conceito e buscando o estabelecimento de medidas de duração confiáveis, cada frase dita passou pela segmentação nestas unidades. $\mathrm{O}$ estabelecimento desta delimitação assegura a adoção de unidades fônicas isomórficas aos efeitos acentuais( ${ }^{(8)}$. As frases estudadas foram divididas em "D/ig/o p/ip/oc/a b/ aix/inho", "D/ig/o c/av/al/o b/aix/inho", "D/ig/o t/ap/et/e b/ aix/inho", sendo que as unidades VV analisadas foram: iG, Up, Uk, Ut, ip, av, ap, oK, aL, eTSH, Ab, Ub , ib, aish, sendo desconsiderados os segmentos inicial ("d") e final ("inhu").

Para a obtenção das medidas acústicas da duração utilizouse o programa Praat, versão 4.3. Respeitamos os seguintes critérios:

a) a medida de duração acústica foi feita a partir da forma da onda e do espectrograma de banda larga do sinal de fala digitalizado, sendo o aspecto visual o principal critério para o corte entre os segmentos acústicos, e o aspecto auditivo usado apenas para confirmação;

b) o corte das unidades foi definido pelo primeiro pulso regular da vogal, com marcação no vale da onda;

c) o primeiro pulso regular se estabeleceu através da uniformidade da amplitude e forma da onda, auxiliado pela ativação da ferramenta formant tracking do software.

Para incrementar a confiabilidade das análises, 50\% dos dados coletados foram aleatoriamente submetidos à confirmação dos valores das medidas acústicas por outro fonoaudiólogo com experiência na área de análise acústica.

Após a coleta dos dados e estabelecimento das medidas acústicas de duração (em milissegundos), os valores obtidos foram tabulados individualmente.

Os dados coletados e tabulados, em números absolutos foram, então, submetidos à análise estatística, através de padronização em Estatística Z (Z-Score), que se baseia na curva normal. Ela mede o quanto um determinado resultado afasta-se da média em unidades de desvio-padrão.

Após a padronização, foi aplicado o Teste ANOVA (Análise de Variância) que é indicado quando se quer comparar três ou mais grupos de informações com nível de mensuração numérica, sendo que as amostras são independentes e/ ou pareadas e deseja-se saber se, em média, os grupos são diferentes. Pode-se testar mais de um efeito com um único modelo.

Para este trabalho, o nível de significância adotado foi de $0,05(5 \%)$.

\section{RESULTADOS}

Os valores de duração acústica das emissões de cada unidade VV estudada (iG”, "Up", “ip", “oK”, "Ab”, Uk”, "av", "aL", “Ub", "Ut", "ap", "eTSH", "Ib", "ajSH”) do grupo de gagos foram comparados com os dos não-gagos. Posteriormente, no grupo de gagos foi feita uma comparação segundo o grau de severidade da gagueira (leve, moderado e severo). 
Tabela 1. Comparação entre os grupos de gagos e não-gagos nas emissões das unidades VV estudadas

\begin{tabular}{|c|c|c|c|c|c|c|c|}
\hline Unidade VV & Grupo & $\mathrm{N}$ & Média & $\mathrm{DP}$ & IC & Valor de $p^{\star \star}$ & Resultado \\
\hline \multirow[t]{2}{*}{$\mathrm{iG}$} & Não-gagos & 18 & $-0,6534$ & 0,1378 & 0,1 & $<0,001^{*}$ & Não-gagos<gagos \\
\hline & Gagos & 54 & 0,2178 & 1,0678 & 0,3 & & \\
\hline \multirow[t]{2}{*}{ Uk } & Não-Gagos & 6 & $-0,5766$ & 0,0858 & 0,1 & $0,009^{*}$ & Não-gagos<gagos \\
\hline & Gagos & 18 & 0,1922 & 1,0929 & 0,5 & & \\
\hline \multirow[t]{2}{*}{ Av } & Não-gagos & 6 & $-0,8005$ & 0,2673 & 0,2 & $0,001^{*}$ & Não-gagos<gagos \\
\hline & Gagos & 18 & 0,2668 & 1,0151 & 0,5 & & \\
\hline \multirow[t]{2}{*}{ AL } & Não-gagos & 6 & $-0,5150$ & 0,0653 & 0,1 & $0,018^{*}$ & Não-gagos<gagos \\
\hline & Gagos & 18 & 0,1717 & 1,1076 & 0,5 & & \\
\hline \multirow[t]{2}{*}{$\mathrm{Ub}$} & Não-gagos & 6 & $-0,4535$ & 0,0585 & 0,1 & $0,036^{*}$ & Não-gagos<gagos \\
\hline & Gagos & 18 & 0,1512 & 1,1203 & 0,5 & & \\
\hline \multirow[t]{2}{*}{ Up } & Não-gagos & 6 & $-0,6982$ & 0,0676 & 0,1 & $0,002^{*}$ & Não-gagos<gagos \\
\hline & Gagos & 18 & 0,2327 & 1,0593 & 0,5 & & \\
\hline \multirow[t]{2}{*}{ Ip } & Não-gagos & 6 & $-0,9727$ & 0,3092 & 0,3 & $<0,001^{*}$ & Não-gagos<gagos \\
\hline & Gagos & 18 & 0,3242 & 0,9379 & 0,4 & & \\
\hline \multirow[t]{2}{*}{ oK } & Não-gagos & 6 & $-0,5602$ & 0,1628 & 0,1 & $0,011^{*}$ & Não-gagos<gagos \\
\hline & Gagos & 18 & 0,1867 & 1,0943 & 0,5 & & \\
\hline \multirow[t]{2}{*}{$A b$} & Não-gagos & 6 & $-0,5355$ & 0,1597 & 0,1 & $0,015^{\star}$ & Não-gagos<gagos \\
\hline & Gagos & 18 & 0,1785 & 1,1002 & 0,5 & & \\
\hline \multirow[t]{2}{*}{ Ut } & Não-gagos & 6 & $-0,5492$ & 0,0533 & 0,0 & $0,012^{*}$ & Não-gagos<gagos \\
\hline & Gagos & 18 & 0,1831 & 1,1001 & 0,5 & & \\
\hline \multirow[t]{2}{*}{ Ap } & Não-gagos & 6 & $-0,5930$ & 0,2805 & 0,2 & $0,010^{*}$ & Não-gagos<gagos \\
\hline & Gagos & 18 & 0,1977 & 1,0790 & 0,5 & & \\
\hline \multirow[t]{2}{*}{ eTSH } & Não-gagos & 6 & $-0,5263$ & 0,1620 & 0,1 & $0,017^{*}$ & Não-gagos<gagos \\
\hline & Gagos & 18 & 0,1754 & 1,1022 & 0,5 & & \\
\hline \multirow[t]{2}{*}{$\mathrm{lb}$} & Não-gagos & 6 & $-0,3877$ & 0,1209 & 0,1 & 0,072 & Não-gagos=gagos \\
\hline & Gagos & 18 & 0,1292 & 1,1304 & 0,5 & & \\
\hline \multirow[t]{2}{*}{ ajSH } & Não-gagos & 18 & $-0,5789$ & 0,2835 & 0,2 & $<0,001^{*}$ & Não-gagos<gagos \\
\hline & Gagos & 54 & 0,1930 & 1,0780 & 0,5 & & \\
\hline
\end{tabular}

Legenda: DP = desvio-padrão; IC = intervalo de confiança. ${ }^{*}$ Valores estatisticamente significantes $(p<0,05) .{ }^{* *}$ Teste t de Student

A Tabela 1 apresenta os resultados da comparação dos grupos de gagos e não-gagos nas emissões das unidades VV estudadas.

A Tabela 2 apresenta os resultados da comparação dos sub-grupos de gagos de acordo com o grau de severidade (leve, moderado e severo) durante as emissões das unidades VV estudadas.

\section{DISCUSSÃO}

No que diz respeito aos aspectos acústicos de duração da fala, as comparações realizadas entre os grupos de gagos e não-gagos, quanto às unidades VV estudadas ("iG", "Up", “ip", “oK”, “Ab”, Uk”, “av”, "aL”, “Ub”, “Ut”, “ap”, "eTSH”, "Ib", "ajSH") demonstradas na Tabela 1 revelam que os gagos utilizaram mais tempo para emitir todas as unidades analisadas (Z-score positivo), sendo a diferença entre essas medidas significante $(\mathrm{p}<0,05)$ em todas as unidades com exceção de "Ib" (p=0,072).

Considerando as unidades por palavra, verificamos que para a palavra "pipoca" os indivíduos gagos utilizaram, em média, 22,03\% mais tempo para emissão quando comparados com os não-gagos. Na emissão da palavra "cavalo", os gagos utilizaram 21,50\% mais tempo e, para "tapete", utilizaram 26,65\% mais tempo que os não-gagos.

Estudos acústicos têm demonstrado que gagos, em média, apresentam duração mais longa das vogais, transições mais lentas entre consoantes e vogais e atraso no início da sonorização de vogais antes de consoantes sonoras ${ }^{(9-12)}$.

Alguns estudos analisaram apenas a duração dos fonemas em palavras, logatomos ou sílabas ${ }^{(8,10-15)}$ enquanto outros buscaram os valores das palavras completas e até das frases $^{(8,14-19)}$.

Tempos maiores de emissão de logatomos em indivíduos gagos na comparação com indivíduos não gagos foram encontrados em alguns estudos ${ }^{(9,17)}$.

Alguns estudos ${ }^{(8-9,11,13-17,20-21)}$ verificaram que os valores médios absolutos de duração da emissão de fonemas e sílabas dos gagos eram maiores que os dos indivíduos nãogagos, como no caso deste estudo. Entretanto, em apenas alguns destes ${ }^{(8-9,14,17,20)}$ as diferenças foram estatisticamente significantes. 
Tabela 2. Comparação entre os sub-grupos de gagos, segundo o grau de severidade nas emissões das unidades VV estudadas

\begin{tabular}{|c|c|c|c|c|c|c|c|}
\hline Unidade VV & Grau de Severidade & $\mathrm{N}$ & Média & DP & IC & Valor de $\mathrm{p}^{\star \star}$ & Resultado \\
\hline \multirow[t]{3}{*}{ iG } & Leve & 18 & $-0,6517$ & 0,1824 & 0,1 & $<0,001^{*}$ & $\mathrm{~L}=\mathrm{M}<\mathrm{S}$ \\
\hline & Moderado & 18 & $-0,4554$ & 0,3487 & 0,2 & & \\
\hline & Severo & 18 & 1,1072 & 0,9977 & 0,5 & & \\
\hline \multirow[t]{3}{*}{ Uk } & Leve & 6 & $-0,5952$ & 0,1756 & 0,2 & $0,003^{*}$ & $\mathrm{~L}=\mathrm{M}<\mathrm{S}$ \\
\hline & Moderado & 6 & $-0,4122$ & 0,3718 & 0,3 & & \\
\hline & Severo & 6 & 1,0074 & 1,1765 & 1,0 & & \\
\hline \multirow[t]{3}{*}{ Av } & Leve & 6 & $-0,3286$ & 0,5342 & 0,5 & $<0,001^{*}$ & $\mathrm{~L}=\mathrm{M}<\mathrm{S}$ \\
\hline & Moderado & 6 & $-0,7653$ & 0,4645 & 0,4 & & \\
\hline & Severo & 6 & 1,0939 & 0,7941 & 0,7 & & \\
\hline \multirow[t]{3}{*}{$\mathrm{AL}$} & Leve & 6 & $-0,5144$ & 0,1962 & 0,2 & $<0,001^{*}$ & $\mathrm{~L}=\mathrm{M}<\mathrm{S}$ \\
\hline & Moderado & 6 & $-0,6352$ & 0,1369 & 0,1 & & \\
\hline & Severo & 6 & 1,1496 & 0,9774 & 0,9 & & \\
\hline \multirow[t]{3}{*}{ Ub } & Leve & 6 & $-0,4767$ & 0,0705 & 0,1 & $0,023^{*}$ & $\mathrm{~L}=\mathrm{M}<\mathrm{S}$ \\
\hline & Moderado & 6 & $-0,3857$ & 0,1358 & 0,1 & & \\
\hline & Severo & 6 & 0,8624 & 1,4258 & 1,2 & & \\
\hline \multirow[t]{3}{*}{ Up } & Leve & 6 & $-0,5379$ & 0,3858 & 0,3 & $<0,001^{\star}$ & $\mathrm{L}=\mathrm{M}<\mathrm{S}$ \\
\hline & Moderado & 6 & $-0,6319$ & 0,2240 & 0,2 & & \\
\hline & Severo & 6 & 1,1698 & 0,8559 & 0,8 & & \\
\hline \multirow[t]{3}{*}{ Ip } & Leve & 6 & $-0,2355$ & 0,7674 & 0,7 & $0,001^{*}$ & $\mathrm{~L}=\mathrm{M}<\mathrm{S}$ \\
\hline & Moderado & 6 & $-0,7752$ & 0,5477 & 0,5 & & \\
\hline & Severo & 6 & 1,0107 & 0,7056 & 0,6 & & \\
\hline \multirow[t]{3}{*}{ oK } & Leve & 6 & $-0,5554$ & 0,3677 & 0,3 & $<0,001^{*}$ & $\mathrm{~L}=\mathrm{M}<\mathrm{S}$ \\
\hline & Moderado & 6 & $-0,5669$ & 0,2785 & 0,2 & & \\
\hline & Severo & 6 & 1,1222 & 0,9593 & 0,8 & & \\
\hline \multirow[t]{3}{*}{$A b$} & Leve & 6 & $-0,3028$ & 0,7119 & 0,6 & $0,023^{*}$ & $\mathrm{~L}=\mathrm{M}<\mathrm{S}$ \\
\hline & Moderado & 6 & $-0,5484$ & 0,2025 & 0,2 & & \\
\hline & Severo & 6 & 0,8512 & 1,2295 & 1,1 & & \\
\hline \multirow[t]{3}{*}{ Ut } & Leve & 6 & $-0,4518$ & 0,1707 & 0,1 & $0,001^{*}$ & $\mathrm{~L}=\mathrm{M}<\mathrm{S}$ \\
\hline & Moderado & 6 & $-0,6264$ & 0,1001 & 0,1 & & \\
\hline & Severo & 6 & 1,0782 & 1,1181 & 1,0 & & \\
\hline \multirow[t]{3}{*}{ Ap } & Leve & 6 & $-0,4566$ & 0,4085 & 0,4 & $0,002^{*}$ & $\mathrm{~L}=\mathrm{M}<\mathrm{S}$ \\
\hline & Moderado & 6 & $-0,5788$ & 0,3617 & 0,3 & & \\
\hline & Severo & 6 & 1,0354 & 1,0787 & 0,9 & & \\
\hline \multirow[t]{3}{*}{ eTSH } & Leve & 6 & $-0,6190$ & 0,1367 & 0,1 & $<0,001^{*}$ & $\mathrm{~L}=\mathrm{M}<\mathrm{S}$ \\
\hline & Moderado & 6 & $-0,5171$ & 0,1394 & 0,1 & & \\
\hline & Severo & 6 & 1,1362 & 1,0159 & 0,9 & & \\
\hline \multirow[t]{3}{*}{$\mathrm{lb}$} & Leve & 6 & $-0,4832$ & 0,1127 & 0,1 & $0,0147^{\star}$ & $\mathrm{L}=\mathrm{M}<\mathrm{S}$ \\
\hline & Moderado & 6 & $-0,4048$ & 0,1004 & 0,1 & & \\
\hline & Severo & 6 & 0,8880 & 1,3980 & 1,2 & & \\
\hline \multirow[t]{3}{*}{ ajSH } & Leve & 18 & $-0,2994$ & 0,6610 & 0,3 & $<0,001^{*}$ & $\mathrm{~L}=\mathrm{M}<\mathrm{S}$ \\
\hline & Moderado & 18 & $-0,5793$ & 0,3214 & 0,2 & & \\
\hline & Severo & 18 & 0,8787 & 1,1444 & 0,5 & & \\
\hline
\end{tabular}

Legenda: $D P=$ desvio-padrão; IC = intervalo de confiança; $L=$ leve; $M=$ moderado; $S=$ severo. * Valores estatisticamente significantes ( $p<0,05)$. ${ }^{\star \star} A N O V A$

Outro estudo ${ }^{(18)}$ comparou a duração da emissão de palavras com composição CVC de indivíduos gagos e não-gagos. Os resultados indicaram que os indivíduos gagos emitiram as palavras de forma mais longa do que o grupo controle, sendo esta diferença estatisticamente significante, assim como neste estudo.

Também considerando as palavras em sua totalidade, um estudo brasileiro ${ }^{(19)}$ demonstrou que os gagos levam mais 
tempo para emitir palavras em tarefas de reconto de história do que não-gagos. Esses achados concordam com os valores absolutos das unidades VV dos gagos desta pesquisa, que são maiores de que os dos não-gagos, com significância em praticamente todos os trechos analisados.

$\mathrm{Na}$ comparação dos subgrupos de gagos (gagos leves $\mathrm{x}$ moderados x severos), em relação às unidades VV estudadas, apresentadas na Tabela 2 podemos observar que os gagos leves e moderados comportam-se semelhantemente e diferem significantemente dos gagos severos, que apresentaram durações mais longas para todas as emissões estudadas, mesmo durante a fluência. Entretanto, exceções foram encontradas quando, no grupo de gagos leves observamos maior tempo para emissão da unidade VV do que no grupo de moderados, como nos casos de "Up", "ip", "oK", "Ab”, "Ut", "ap", “av", "aL" e "aish".

$\mathrm{Na}$ literatura não foram encontradas referências sobre a diferença entre a duração das emissões dos indivíduos gagos segundo o grau de severidade da gagueira. Entretanto, um estudo $^{(22)}$ indicou que os indivíduos com gagueira de grau moderado/severo apresentaram tempo de movimentação de mandíbula mais longo, em tarefa de abertura e fechamento mandibular sem feedback visual, do que os de grau muito leve/ leve. O tempo mais longo para a realização do movimento observado neste estudo, pode resultar em uma duração mais longa nas emissões de fala que envolvam qualquer ação desse articulador.

Embora os autores tenham utilizado metodologia diferente no cálculo das medidas acústicas, verificaram, em um estudo ${ }^{(15)}$ de 1986, que, assim como neste estudo, os indivíduos gagos levavam mais tempo para emitir frases pré-estabelecidas, em situação de fluência, quando comparados com os indivíduos não-gagos, ou seja, apresentavam produções mais longas das unidades que compunham o corpus das frases.

As medidas acústicas de duração anômalas observadas nos indivíduos gagos deste estudo muito provavelmente estão associadas à questão da duração da sílaba na palavra e à da ordem sequencial dos espaços fonéticos.

Explicações para tal fato podem ser encontradas no modelo já proposto ${ }^{(23)}$. Segundo este modelo, para que a fala seja fluente, no que diz respeito à temporalidade, ao menos dois sistemas operacionais neurais devem estar equilibrados antes de a mensagem chegar ao córtex motor. O primeiro sistema é o simbólico, que se relaciona às questões cognitivas, linguísticas, fonológicas (ritmo e entonação) e fragmentais (inicio e fim) e é responsável por determinar a forma e o conteúdo da mensagem, ou seja, a segmentação. Já o segundo sistema é o de sinais, que se relaciona a prosódia (frequência, intensidade, duração) e determina a duração da sílaba na palavra e a ordem de sequência dos espaços fonéticos. Quando a segmentação e a ordenação operam em equilíbrio a fala é fluente. Quando há desequilíbrio, o fluxo da fala é rompido gerando as rupturas ou disfluências.

Deste modo, podemos hipotetizar que as medidas de duração diferentes entre gagos e não-gagos, observadas neste estudo, em situações de fala fluente, evidenciam um desequilíbrio no sistema de sinais.

Considerando que a fala é uma tarefa de controle motor rápido e que, provavelmente, nos gagos o tempo de ativação e seleção fonêmica que compõe o plano fonético para a fala seja aumentado e a atividade motora mais lentificada ${ }^{(24)}$. Sendo assim, iniciar rapidamente uma emissão ou falar rapidamente exige esforço excessivo desse sistema, gerando as rupturas. $\mathrm{Na}$ fala fluente, a necessidade dos gagos de um maior tempo de ativação e seleção fonêmica geraria uma duração de fala mais longa do que os de não-gagos.

A diferença estatisticamente significante encontrada nas emissões no grupo de gagos, no que diz respeito às unidades $\mathrm{VV}$, pode ser justificada por um atraso no processamento motor que ocorre entre a entrada do sinal sensorial e a saída motora, indicando déficit no controle motor da fala nos gagos ${ }^{(12)}$. Alem disso, podemos suspeitar que a inabilidade dos gagos para processar sinais neurais para efetuar os movimentos precisos e velozes da conversação normal, especialmente quando estão sob a pressão do planejamento de uma sentença complexa ou competindo com outros falantes, também esteja relacionada com as medidas de duração maiores observadas nos gagos ${ }^{(12)}$.

O controle motor da fala exige coordenação de processos neurais e mecanismos sensório-motores, que possibilite um discurso fluente ${ }^{(25-27)}$. Na gagueira, podemos observar incoordenações ou rupturas dos movimentos respiratórios, fonatórios e/ou articulatórios, caso ocorra alguma alteração em algum nível deste controle ${ }^{(28)}$. Já, em situação de fluência, os gagos podem manifestar uma lentidão na emissão, com consequente aumento da duração acústica e da taxa de elocução devido ao déficit processual.

Aspectos relacionados ao início da fonação e da articulaçãa $^{(11,23,29)}$ indicam que os gagos apresentam maior lentidão quando comparados aos não-gagos.

A lentidão geral nos movimentos de fala observada nos indivíduos gagos, possivelmente de base sensorial, expressa-se durante a fala fluente, de maneira aparentemente harmoniosa, ou seja, a duração maior da emissão reflete os aspectos básicos de um mecanismo subjacente à desordem ou uma estratégia motora preferencialmente utilizada pelos gagos para evitar ou minimizar os episódios de disfluência ${ }^{(28)}$.

As diferenças entre os parâmetros de duração acústica aqui verificadas (unidades VV isoladas e taxas de elocução) tornam evidente a importância destas na avaliação, diagnóstico e terapia dos gagos ${ }^{(12,30)}$.

Tendo em vista a verificação de maiores valores de emissão das unidades VV e consequentemente de menores taxas de elocução, devemos utilizar como estratégia terapêutica a diminuição da velocidade de fala, não só para simplificar a produção possibilitada por uma programação articulatória mais suave de cada unidade, mas também para estabilizar o sistema e compensar a instabilidade inerente do mecanismo motor de fala dos indivíduos gagos.

\section{CONCLUSÃO}

A análise de parâmetros de duração acústica de unidades delimitadas por onsets vocálicos consecutivos demonstrou que os sujeitos gagos diferem dos sujeitos não-gagos e indicou diferenças importantes entre os graus de severidade da gagueira.

Os achados evidenciam que há necessidade de estudos mais 
aprofundados, principalmente no que diz respeito à comparação quanto aos graus de severidade da gagueira, que possam auxiliar na compreensão desta patologia complexa e de como o processo de programação motora difere nestes sujeitos. O uso de dados acústicos objetivos também poderá auxiliar no estabelecimento de parâmetros pré e pós-terapêuticos. Além disso, estudos que associem os aspectos da duração acústica com exames de imagem, eletromiografia, manipulação de parâmetros auditivos, etc., podem colaborar para explicar o envolvimento dos fatores de programação e seleção motora, mobilidade muscular, entre outros.

\section{AGRADECIMENTOS}

À Coordenação de Aperfeiçoamento de Pessoal de Nível Superior (CAPES) pelo financiamento concedido para a realização desta pesquisa.

\begin{abstract}
Purpose: To compare utterance duration of fluent speech of adults with different degrees of stuttering on a phrase repetition task. Methods: Six adult stutterers, with severity degrees varying from mild to severe, were matched to individuals with no communication problems. The corpus was constituted by the words "horse", "popcorn" and "carpet" introduced into the vehicle-phrase "I say...... quietly". Each phrase was uttered by the researcher and repeated aloud three times by the participants. The speech sample was recorded on a computer, and phrases containing disfluencies were rejected. After that, measurements of the acoustic duration of each sample were carried out using the software Praat 4.3. The phrases were divided into segments delimited by two consecutive voice onsets. Data were tabulated and statistically analyzed using t-test and Analysis of Variance (ANOVA). Results: Stutterers took longer to produce all the units analyzed, except for the unit [ib]. The comparison among different stuttering severities showed that mild and moderate stutterers had similar duration measures, which was significantly different from severe stutterers. Conclusion: The analysis of acoustic duration parameters of units delimited by consecutive voice onsets demonstrated that stutterers differed from non-stutterers; moreover, these measures were able to differentiate among stuttering severity degrees.
\end{abstract}

Keywords: Articulation disorders; Speech disorders; Stuttering; Speech acoustics; Speech production measurement; Sound spectrography

\title{
REFERÊNCIAS
}

1. Van Riper C. The nature of stuttering. 2nd ed. Englewood Cliffs (NJ): Prentice-Hall; 1982.

2. Andrade CR. Abordagem neurolinguística e motora da gagueira. In: Ferreira LP, Befi-Lopes DM, Limongi SC, organizadores. Tratado de fonoaudiologia. São Paulo: Roca; 2004. p. 1001-16.

3. Schiefer AM. Abordagem psicolinguística da fluência. In: Ferreira LP, Befi-Lopes DM, Limongi SC, organizadores. Tratado de fonoaudiologia. São Paulo: Roca; 2004b. p. 1035-9.

4. Kent RD, Read C. The acoustic analyses of speech. San Diego: Singular Publishing Group; 1992.

5. Barbosa PA. Incursões em torno do ritmo da fala. São Paulo: FAPESP; 2006.

6. Albano EC. O gesto e suas bordas: esboço de fonologia acústicoarticulatória do português brasileiro. São Paulo: FAPESP; 2001.

7. Riley GD. A stuttering severity instrument for children and adults, SSI-3. 3rd ed. Austin: ProEd; 1994.

8. Colaccico FB, Schiefer AM, Osborn, E. Medidas Acústicas de duração da fala de indivíduos gagos e não-gagos. Fono Atual. 2005;8(31):26-30.

9. Di Simoni FG. Letter: Preliminary study of certain timing relationships in the speech of stutterers. J Acoust Soc Am. 1974;56(2):695-6.

10. Hillman RE, Gilbert HR. Voice onset time for voiceless stop consonants in the fluent reading of stutterers and nonstutterers. J Acoust Soc Am. 1977;61(2):610-11.

11. Zimmermann G. Articulatory dynamics of fluent utterances of stutterers and nonstutterers. J Speech Hear Res. 1980;23(1):95-107.

12. Guitar B. Stuttering: an integrated approach to its nature and treatment. 3rd ed. Baltimore (MD): Lippincott Williams Wilkins; 2006.

13. Metz DE, Conture EG, Caruso A. Voice onset time, frication, and aspiration during stutterers' fluent speech. J Speech Hear Res. 1979;22(3):649-56.
14. Zebrowski PM, Conture EG, Cudahy EA. Acoustic analysis of young stutterers' fluency: preliminary observations. J Fluency Disord [Internet] 1985 [cited 2008 Jun 16];10(3):173-92. Available from: http:// www.sciencedirect.com/science?_ob=ArticleURL\&_udi=B6T8H45RC9WDB\&_user $=10 \&$ \&coverDate $=09 \% 2 \mathrm{~F} 30 \% 2 \mathrm{~F} 1985 \&$ rdoc $=1 \&$ _ $\mathrm{fmt}=\mathrm{high} \& \_$orig $=$browse\&_sort $=\mathrm{d} \& \mathrm{view}=\mathrm{c} \& \_\mathrm{acct}=\mathrm{C} 000050221 \&$ version=1\&_urlVersion=0\&_userid=10\&md5=181fe $908196040294 \mathrm{e} 6$ efb8b56945834

15. Healey EC, Ramig PR. Acoustic measures of stutterers' and nonstutterers' fluency in two speech contexts. J Speech Hear Res. 1986;29(3):325-31.

16. Oliveira CM, Trindade IE, Yamashita RP, Trindade Junior AS. Aspectos temporais da fala fluente de gagos: avaliação aerodinâmico-acústica. Acta AWHO. 2000;19(1):42-8.

17. Colaccico FB, Arcuri CF, Osborn E, Schiefer AM. Caracterização dos aspectos de duração da fala de indivíduos fluentes e gagos. Rev Soc Bras Fonoaudiol. 2006;11(3):158-62.

18. Blomgren, M. Stability of segment durations in stutterers' and nonstutterers' speech: task complexity effects. In: IALP $25^{\text {th }}$ World Congress; 2001 Jan 31; Montreal. Montreal: International Association of Lean Practitioners; 2001.

19. Arcuri CF, Chiari BM, Osborn E, Schiefer AM. Medidas acústicas de duração no reconto de história em indivíduos gagos. Acta ORL [Internet] 2006 [citado 2008 Jun 16];24(3):113-16. Available from: http://www.actaorl.com.br/detalhe_artigo.asp?id=80

20. Borden GJ, Kim D, Spiegler K. Acoustics of stop consonant-vowel relationships during fluent and stuttered utterances. J Fluency Disord. 1987;12(3):175-84.

21. Peters HF, Boves L. Coordination of aerodynamic and phonatory processes in fluent speech utterances of stutterers. J Speech Hear Res. 
1988;31(3):352-61.

22. Archibald LM, De Nil LF. The relationship between stuttering severity and kinesthetic acuity for jaw movements in adult stutterers. J Fluency Disord. 1999;24(1):25-42.

23. Perkins WH, Kent RD, Curlee RF. A theory of neuropsycholinguistic function in stuttering. J Speech Hear Res. 1991;34(4):734-52.

24. Ludlow CL, Loucks T. Stuttering: a dynamic motor control disorder. J Fluency Disord. 2003;28(4):273-95.

25. Guenther FH. Speech sound acquisition, coarticulation, and rate effects in a neural network model of speech production. Psychol Rev. 1995;102(3):594-621.

26. Bailly G. Learning to speak. Sensori-motor control of speech movements. Speech Commun [Internet] 1997 [cited 2008 Jun 16] ;22(23):251-67. Available from: http://cat.inist.fr/?aModele=afficheN\&cpsi $\mathrm{dt}=2095231$.
27. Kent RD. Research on speech motor control and its disorders: a review and prospective. J Commun Disord. 2000;33(5):391-427; quiz 428.

28. Max L, Guenther FH, Gracco VL, Ghosh SS, Wallace ME. Unstable or insufficiently activated internal models and feedback-biased motor control as sources of dysfluency: a theoretical model of stuttering. Contemp Issues Commun Sci Disord [Internet] 2004 [cited 2008 Jun 16];31:105-22. Available from: http://www.asha.org/NSSLHA/ publications/s04cicsdabstracts.htm.

29. Max L, Caruso AJ, Gracco VL. Kinematic analyses of speech, orofacial nonspeech, and finger movements in stuttering and nonstuttering adults. J Speech Lang Hear Res. 2003;46(1):215-32.

30. Brosch S, Häge A, Johannsen HS. Prognostic indicators for stuttering: the value of computer-based speech analysis. Brain Lang. 2002;82(1):75-86. 